January E. Gelera, M.D.

Norberto V. Martinez, M.D.

Department Of Otorhinolaryngology Head and Neck Surgery

University of Santo Tomas Hospital

Manila, Philippines

\section{Undifferentiated Nasopharyngeal Malignancy with Immunohistochemical Features of Diffuse Large B Cell Lymphoma and Undifferentiated Carcinoma: A Collision Tumor?}

\author{
ABSTRACT \\ carcinoma.

\section{Methods:} \\ Design: Case Report \\ Setting: Tertiary Private University Hospital \\ Patient: One
}

Objective: To describe an unusual presentation of undifferentiated nasopharyngeal malignancy with immunohistochemical features of both diffuse B-cell lymphoma and undifferentiated

Results: A 49-year-old female whose initial nasopharyngeal biopsy interpretation was diffuse large B-cell lymphoma underwent three cycles of Rituximab, Cyclophosphamide, Hydroxydaunomycin, Oncovin and Prednisone (R-CHOP). Post-chemotherapy Computed Tomography (CT) scan of the nasopharynx revealed no change in tumor size or appearance. Repeat nasopharyngeal (NP) biopsy findings suggested an epithelial tumor lineage or post-chemotherapy reactive mucosal epithelial cells. No residual lymphoma was noted and immunostaining was positive for cytokeratin. The patient underwent 35 fractions of radiotherapy. Re-evaluation by Magnetic Resonance Imaging (MRI) with contrast after four months showed significant tumor shrinkage. Repeat NP biopsy revealed necrotic tissues with foci of high-grade squamous cell carcinoma. Two months after the biopsy, repeat MRI with contrast of the nasopharynx and neck showed increase in the bulk of the nasopharyngeal tumor with inferior extension to the level of the orophaynx and possible contralateral involvement. A nasopharyngectomy via left maxillary swing was performed and the final histopathology was undifferentiated carcinoma.

Conclusion: Undifferentiated malignancies of the nasopharynx may contain lymphoma or carcinoma and rarely, both lineages in coexistence. In such cases, the possibility of a collision tumor should be considered. Immunohistochemical distinction is important for treatment and prognostication.

Correspondence: Dr. January E. Gelera

University of Santo Tomas Hospital

España Street, Sampaloc, Manila 1015

Philippines

Phone: 7313001 local 2224

Email:janjanmd@yahoo.com

Reprints will not be available from the authors

The authors declared that this represents original material that is not being considered for publication or has not been published or accepted for publication elsewhere, in full or in part, in print or electronic media; that the manuscript has been read and approved by all the authors, that the requirements for authorship have been met by each author, and that each author believes that the manuscript represents honest work.

Disclosures: The authors signed disclosures that there are no financial or other (including personal) relationships, intellectual passion, political or religious beliefs, and institutional affiliations that might lead to a conflict of interest.
Keywords: nasopharyngeal carcinoma, undifferentiated tumor, undifferentiated carcinoma, nasopharyngeal lymphoma, collision tumors, immunohistochemistry, Diffuse large B-cell lymphoma, secondary malignancy

The term "undifferentiated tumor" has been used in reference to a heterogeneous group of tumors with little or no evidence of differentiation. These tumors lack evidence of lineage differentiation on the basis of routine light microscopic morphology, and immunohistochemical evaluation is employed to differentiate between malignant lymphoma - (LCA) lymphoid lineage, nasopharyngeal carcinoma - (cytokeratin) epithelial lineage, rhabdomyosarcoma - (vimentin) messenchymal lineage and yolk sac tumor - (a-fetoprotein) germ cell lineage. ${ }^{1}$ 


\section{CASE REPORTS}

Distinction between these lineages is important for treatment and prognostication. For an undifferentiated tumor, the diagnosis of lymphoma generally predicts a better clinical outcome than a diagnosis of carcinoma. ${ }^{2}$

On the other hand, the occurrence of collision tumors in the human body is extremely rare. The term refers to coexistent but histologically independent tumors occurring in the same organ. The biological behavior of these tumors is difficult to ascertain and clinical awareness and recognition will oftentimes determine the final outcome in terms of disease-free survival time.

This case report will illustrate the significance of immunohistochemistry in identifying the true identity of undifferentiated tumors and the possibility of collision tumors of carcinoma and lymphoma of the nasopharynx or development of secondary malignancy.

\section{CASE REPORT}

A 49-year-old female consulted due to one-year progressive hearing loss, aural fullness and occasional tinnitus in the left ear with weight loss. Otoscopy revealed otitis media with effusion and nasal endoscopy showed a nasopharyngeal mass, both on the left. No cervical lymph node enlargement was appreciated. Nasopharyngeal (NP) biopsy was interpreted as undifferentiated malignancy. (Figure 1) Immunohistochemical evaluation was positive for lymphoma and B-cell lineage (Figure 2, 3) and negative for T-cell lineage and carcinoma. (Figure 4, 5) The results were consistent with Diffuse Large B-Cell Lymphoma (DLBCL) and three cycles of Rituximab, Cyclophosphamide, Hydroxydaunomycin, Oncovin and Prednisone (R-CHOP) were started.

After three months of treatment repeat contrast-enhanced CT scan showed no apparent change in tumor size (Figure 6) and a second NP biopsy was read as undifferentiated carcinoma. Immunostaining was positive for cytokeratin (Figure 7) and negative for LCA. (Figure 8) Management was shifted to concurrent chemoradiotherapy (CRT) of high-dose 3-weekly Cisplatin and Simultaneous Integrated Boost Intensity Modulated Radiotherapy (SIB IMRT) of 70Gy in 35 fractions.

Four months after concurrent CRT, contrast MRI showed decrease in nasopharyngeal tumor size and absence of cervical adenopathies. (Figure 9) A third NP biopsy revealed necrotic tissues with foci of highgrade squamous cell carcinoma. Unfortunately, the patient was lost to follow-up until two months after, when another contrast MRI showed

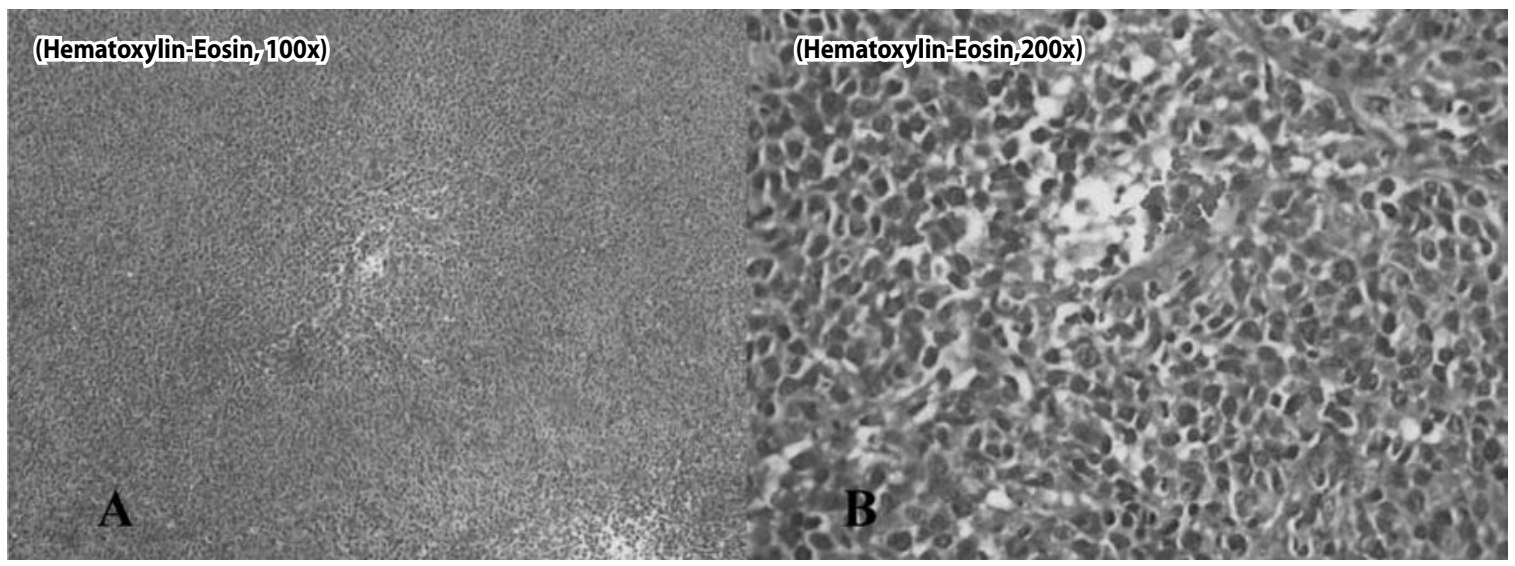

Figure 1. Hematoxylin - Eosin stain. A. 100x Magnification B. 200x Magnification. Microsections disclose NP tissue with a malignant neoplasm composed of polyhedral cell nests infiltrating the lymphoid stroma. Tumor cells exhibit round to ovoid hyperchromatic nuclei, prominent nycleoli and scanty cytoplasm.

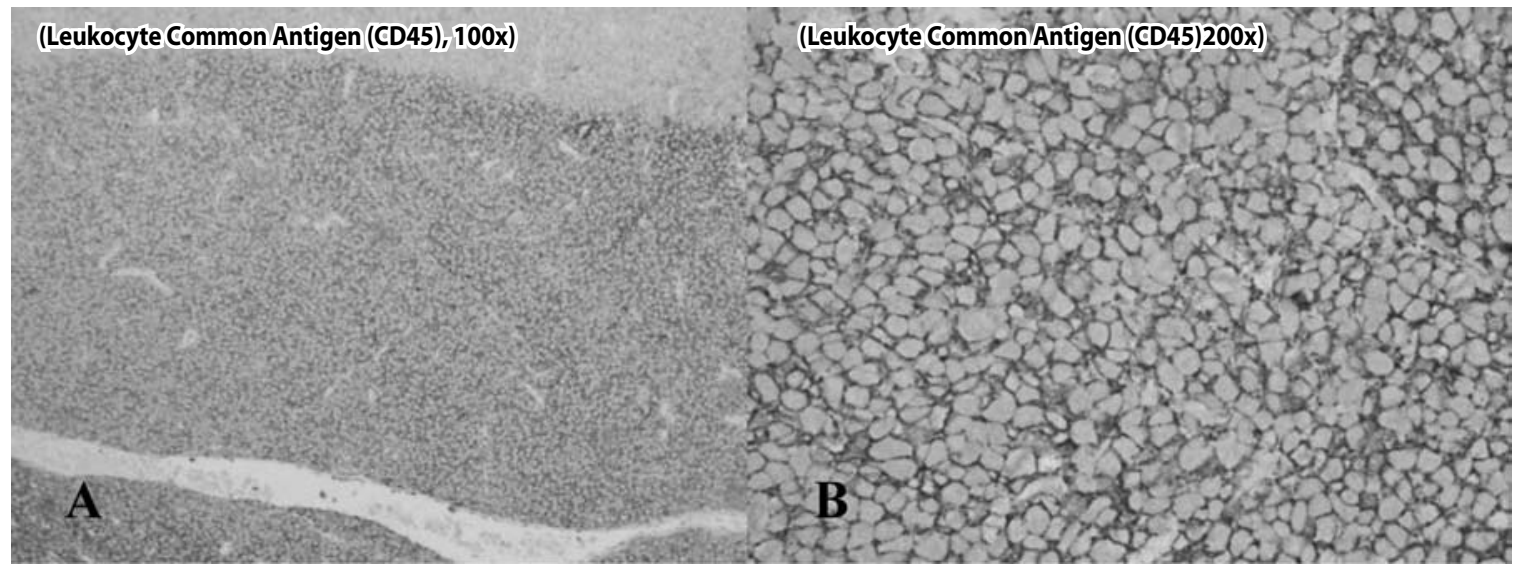

Figure 2. Leukocyte Common Antigen (CD45). A. 100x Magnification B. 200x Magnification. Positive in sheets of large lymphoid 


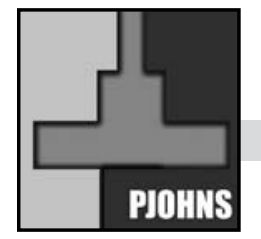

\section{CASE REPORTS}

Philippine Journal Of Otolaryngology-Head And Neck Surgery

Vol. 27 No. 1 JANUARY - JuNE 2012

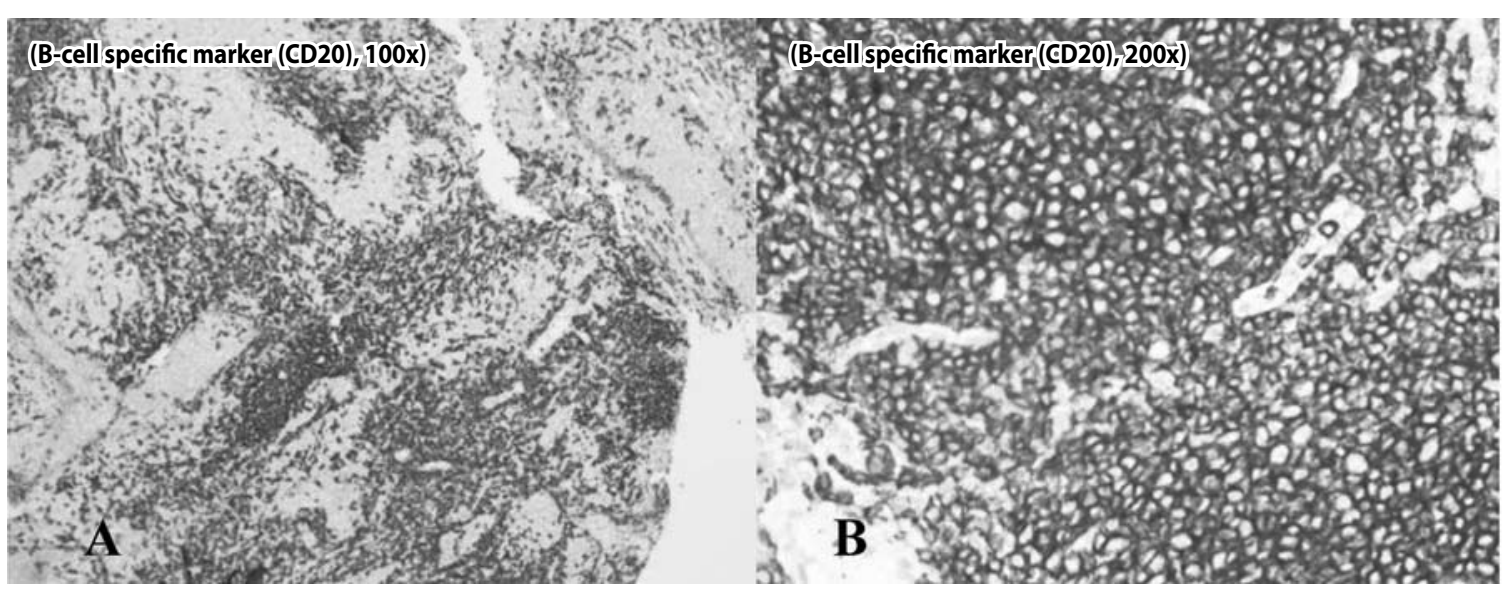

Figure 3. B-cell specific marker (CD20). A. 100x Magnification B. 200x Magnification. Positive in sheets of large B-cells

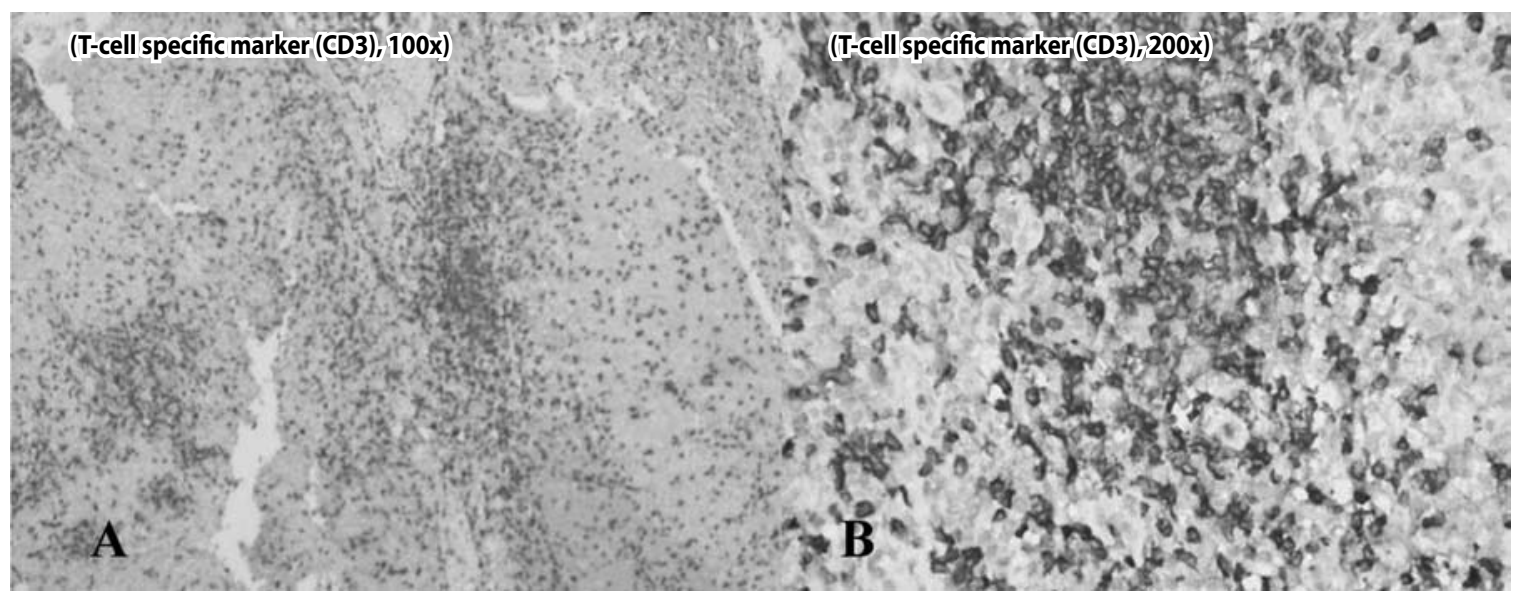

Figure 4. T-cell specific marker (CD3). A. 100x Magnification B. 200x Magnification. Negative in sheets of large cells; positive in scattered small mature T-lymphocytes

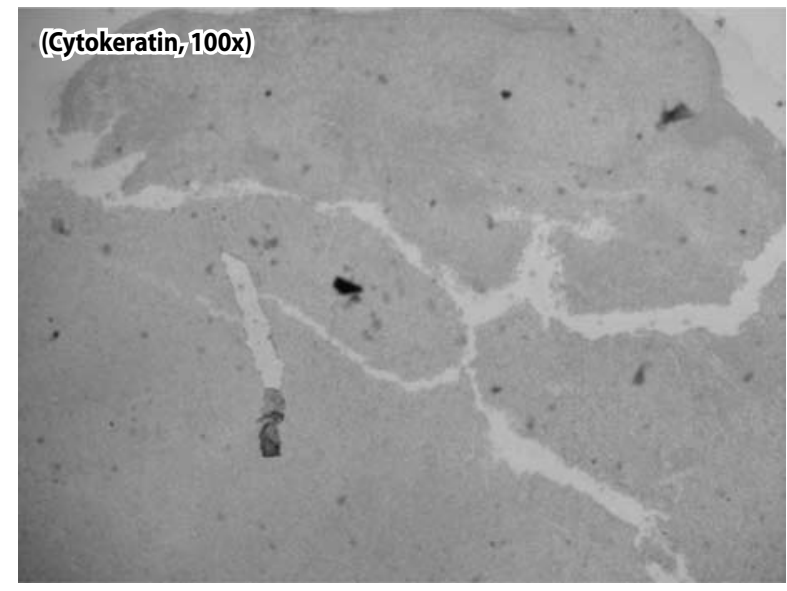

Figure 5. Cytokeratin 100x Magnification. Epithelial cells staining negatively for cytokeratin

increase in the bulk of the nasopharyngeal tumor on the left with inferior extension to the level of the orophaynx and possible contralateral involvement. (Figure 10) Based on the consensus at a multidisciplinary meeting, a nasopharyngectomy via left maxillary swing was performed, and the final histopathology report was undifferentiated carcinoma.

\section{DISCUSSION}

The first NP biopsy histophathology reading was an undifferentiated malignancy. The term undifferentiated malignancy refers to a heterogenous group of tumors with little or no evidence of differentiation. The term undifferentiated also refers to tumors lacking evidence of lineage differentiation on the basis of routine light microscopic morphology. The characterization of epithelial, mesenchymal, melanocytic or hematopoeitic origin is crucial for treatment planning and prognostication., ${ }^{3,4}$

Undifferentiated malignancies of the nasopharynx may resemble large cell or immunoblastic types of malignant lymphoma and may be distinguished from a carcinoma by a positive immunoreactivity for LCA and negative reaction for cytokeratin. ${ }^{4,5}$ This identification is important in undifferentiated tumors because the diagnosis of lymphoma generally predicts a better clinical outcome than diagnosis of carcinoma. ${ }^{5}$ Studies have demonstrated a greater incidence of lymphomas in cases of undifferentiated nasopharyngeal malignancy. Gatter et al. further illustrated that when a pathologist is uncertain about the origin of a neoplasm, the tumor is likely (by a factor of 3 to 1) to be lymphoid in origin. ${ }^{6}$ In cases where a diagnosis of carcinoma is 
CASE REPORTS

Philippine Journal Of Otolaryngology-Head And Neck Surgery

Vol. 27 No. 1 JANUARY - JUNE 2012
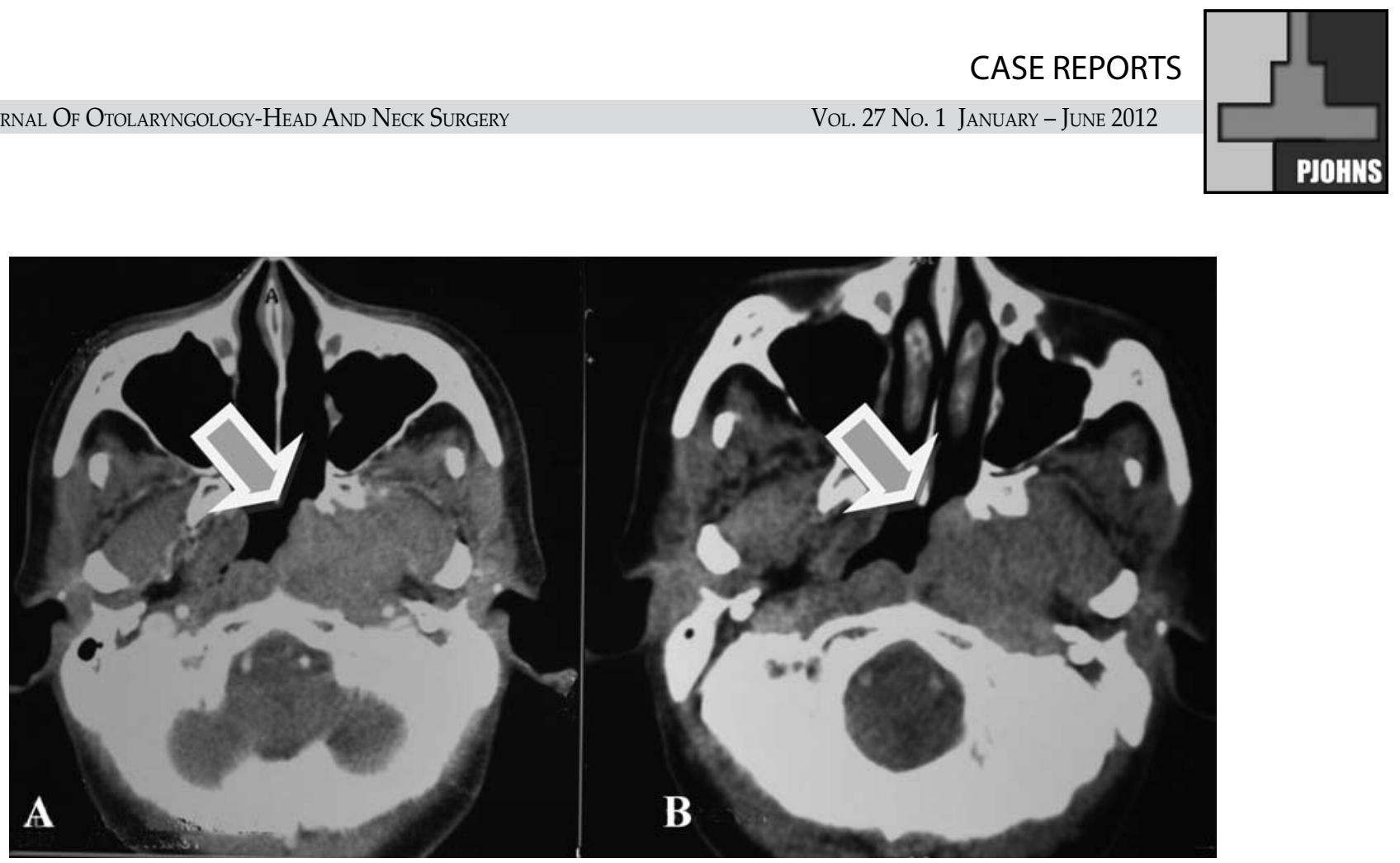

Figure 6. A. September 2008. B. January 2009. No apparent interval change in size appearance and configuration compared with the previous CT.

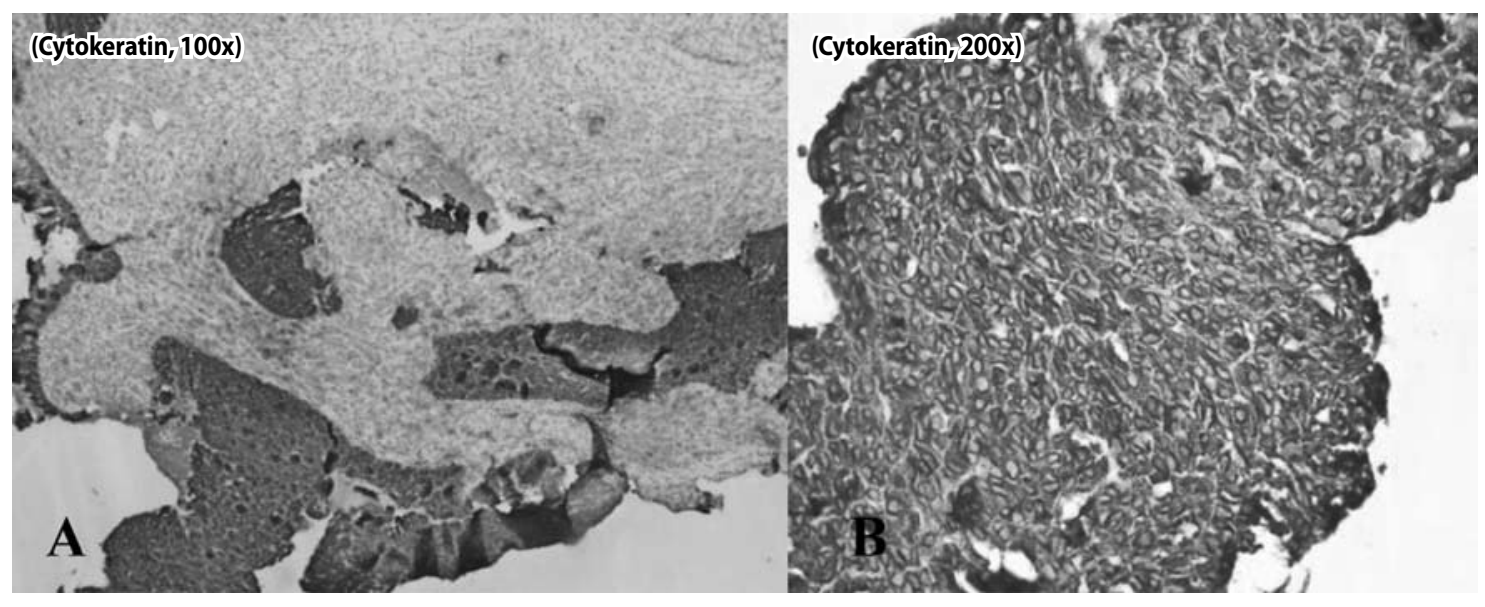

Figure 7. Repeat NP biopsy Cytokeratin A. 100x Magnification. B. 200x Magnification. Epithelial cells stained positively for cytokeratin indicating a carcinoma lineage.

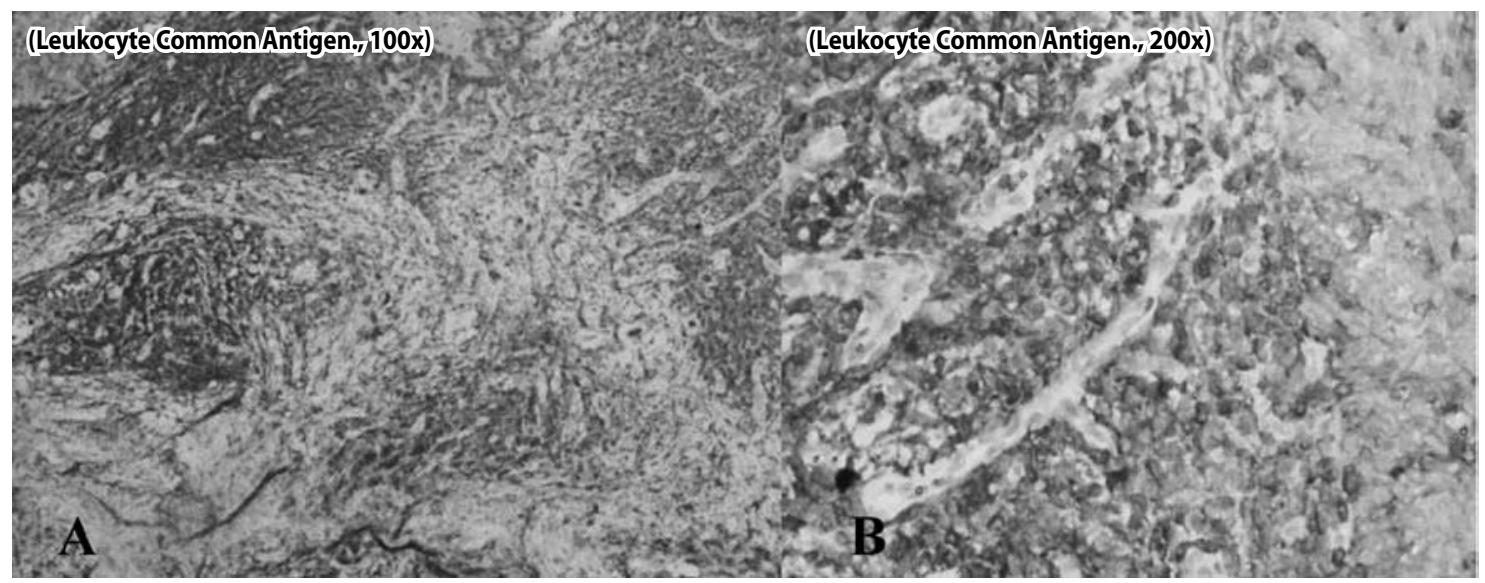

Figure 8. Repeat NP biopsy. Leukocyte Common Antigen. A. 100x Magnification. B. 200x Magnification. Negative immunostainingg of the cytoplasm indicates negativity for lymphoma lineage. 


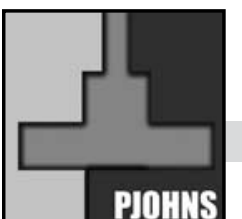

\section{CASE REPORTS}

Philippine Journal Of Otolaryngology-Head And Neck Surgery

Vol. 27 No. 1 JANUARY - JUNE 2012

PJOHLS

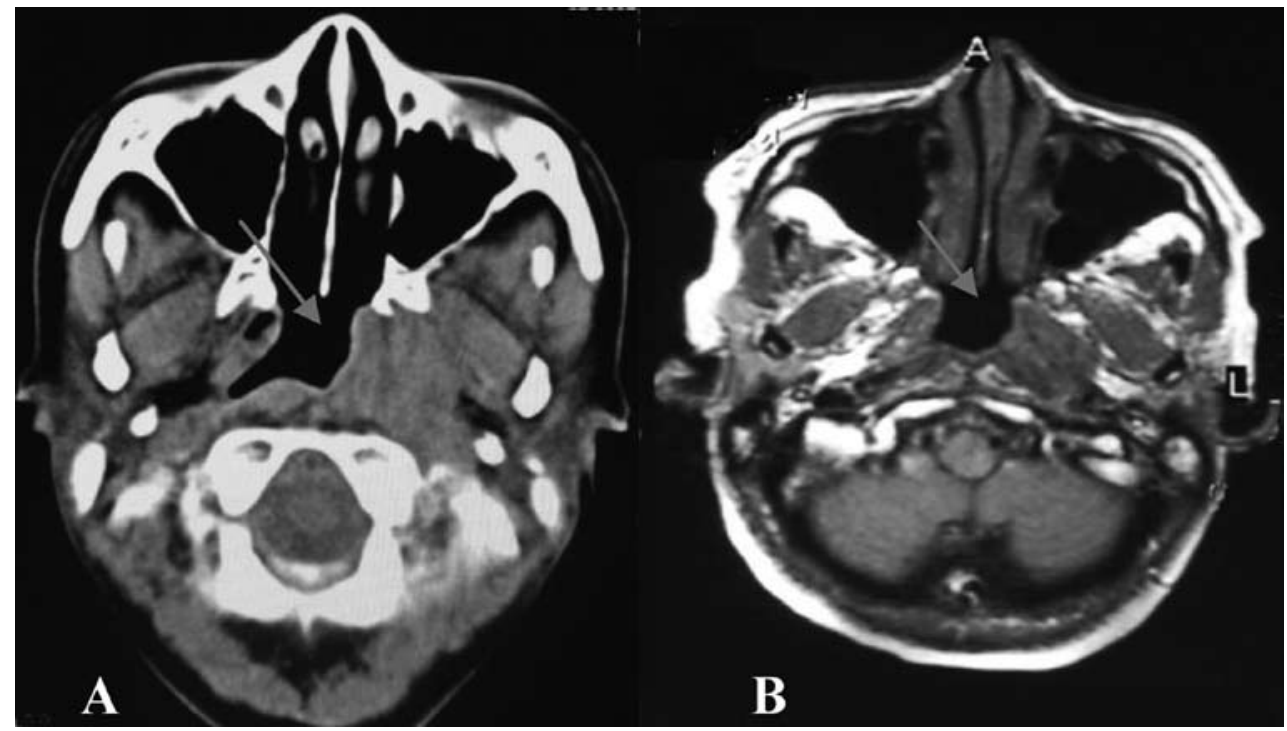

Figure 9. A. CT July 2009 scan B. MRI July 2009. Four months after completion of radiotherapy a significant decrease in the size of left nasopharyngeal mass was observed.

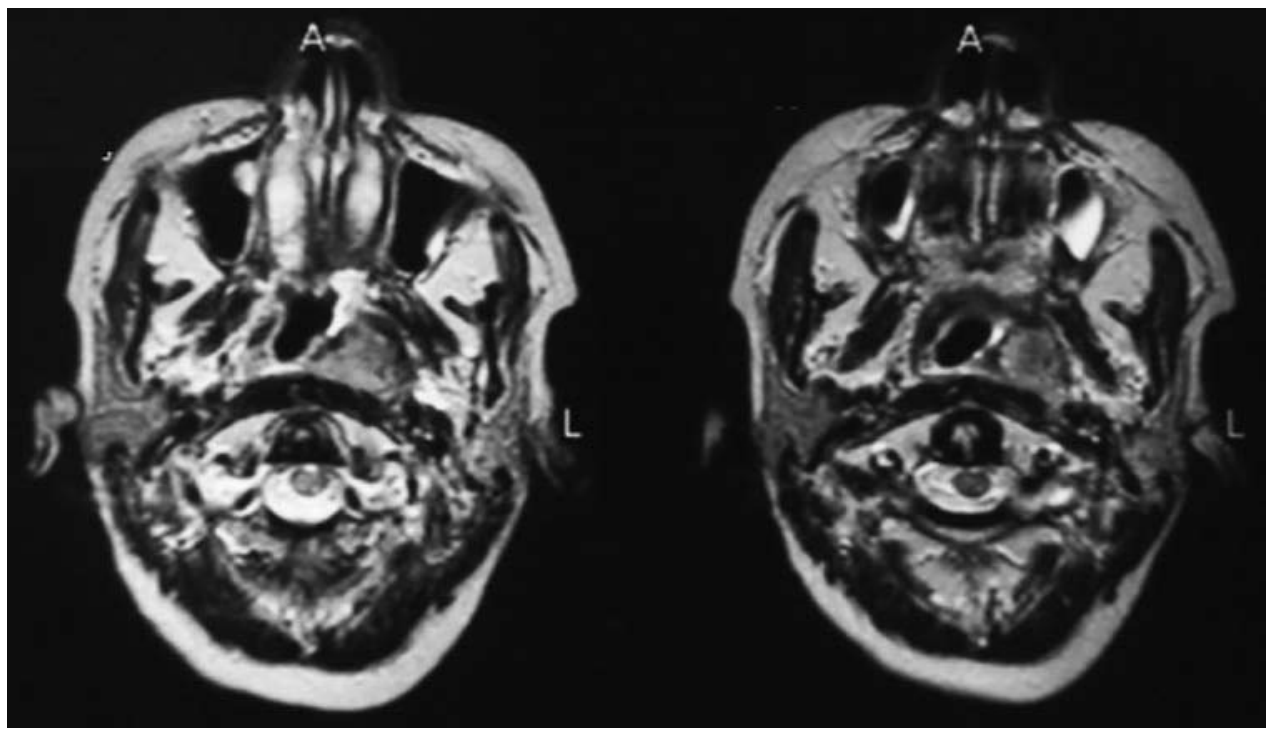

Figure 10. MRI with contrast June 2010. increase in the bulk of the nasopharyngeal tumor on the left with inferior extension to the level of the orophaynx and possible contralateral involvement.

favored, the frequency of it being a lymphoma is higher., ${ }^{6,7}$

Immunostaining was done in this case to reveal the true identity of the tumor. The specimens from the first NP biopsy were immunopositive for LCA and CD20 while cytokeratin and CD3 were immunonegative. LCA antibody for lymphoma and keratin antibody for carcinoma are useful immunohistochemical tools in the differential diagnosis of undifferentiated neoplasms. ${ }^{7.8}$ Non-Hodgkins lymphoma of B and T cell types are immunoreactive to LCA in $93 \%$ to $100 \% .{ }^{7.8}$

Prior to the start of chemotherapy, numerous laboratory and metastatic evaluations all indicated that the primary tumor was present only in the nasopharynx. Following R-CHOP every three weeks for three cycles, repeat CT scans of the nasopharynx and neck showed no evidence of tumor regression or change in appearance and configuration.

Could this have been a residual mass or another tumor entity? Positron emission tomography combined with a radiotracer 18F-fluoro-2deoxyglucose (PET-FDG) would have been very useful in detecting the activity of this residual tumor because malignant cells have increased glycolytic activity. ${ }^{9}$ In this case, a tissue sample was still a reasonable choice because it can correctly identify the tumor and is cost-effective. 


\section{CASE REPORTS}

Could this have been a secondary malignancy after chemotherapy? The success in combating non-Hodgkin's lymphoma with R-CHOP during the last decade cannot be overemphasized. This successful treatment resulted in long-term survival of patients, putting them at risk for late sequelae, particularly secondary malignancy. A secondary malignancy is a new cancer that occurs in an individual as a result of previous treatment with radiotherapy and chemotherapy that may occur months or years from initial treatment. Sacchi et al., estimated the average development of secondary malignancy at 51 months. Among 1280 patients with DLBCL eligible for their study, 48 (3.8\%, crude rate 7.6 per 1000 person-years) developed secondary malignancy. Of these, 35 developed solid tumors such as colorectal (8), lung (8) and others (19) while the rest were hematologic malignancies (8)..$^{10}$ Notably, none developed nasopharyngeal malignancy. Our case is unlikely to have been a secondary malignancy because any mitotic changes caused by initial treatment would have taken months or years to manifest. Moreover, the first and second NP biopsy results were reviewed by pathologists from three different tertiary hospitals in Metro Manila and the reviews were consistent with our previous findings.

Now, could this still be a lymphoma with an unusual immunohistochemistry result? Lasota et al. reported five cases of clinically aggressive, keratin-positive malignant lymphomas of B-cell type that showed unusual immunophenotypes. Two of the cases lacked reactivity to LCA while three were immunopositive for cytokeratin. All five were documented as B-cell lymphoma on the basis of polymerase chain reaction suggesting that some B-cell lymphomas can have confusing immunophenotypes with keratin positivity and leukocyte antigen negativity." Such a possibility may have been considered given the increased nasopharyngeal tumor size after CRT treatment. Molecular genetic studies would have been a logical next step, but the multidisciplinary consensus was to treat the persistence as radioresistance, hence the nasopharyngectomy.

Could this have been an immunohistochemistry error from the beginning? We believe this case underwent a reasonable immunohistochemistry panel. The tissue samples were routinely fixed with $10 \%(\mathrm{v} / \mathrm{v})$ phosphate-buffered formalin embedded in paraffin and cut at 5 um. Standard immunohistochemistry staining techniques were performed with a Dako EnVision Flex ${ }^{\mathrm{TM}}$ kit (Dako Cytomation, Denmark). Sources of error in immunohistochemistry include denatured antibody, loss of antigen due to poor fixation, antigen level below detection, cross-reactivity and non-specific binding of antibody. Our institution followed strict guidelines to avoid such errors, including preliminary evaluation of some slides to ensure optimal quality. Furthermore, the first NP biopsy was processed and reviewed by two experienced pathologists with one being a hemapathologist. These slides were subsequently reviewed at three tertiary private institutions in Metro Manila and the results were the same.

Could this have been a case of collision tumor on the same site? Reports of co-existence are not uncommon because the risk of multiple malignancies in the head and neck region are reported at 2-11 per cent. ${ }^{21}$ Collision tumors represent a coexistence of two adjacent but histologically-different malignant neoplasms occurring in the same organ without histological admixture or an intermediate cell population zone. ${ }^{13}$ Theories include: 1 ) "chance accidental meeting" of two primary tumors; 2) presence of the first tumor alters the microenvironment facilitating the development of the second primary tumor or seeding of metastatic tumor cells; 3 ) simultaneous proliferation of two different cell lines. ${ }^{23}$ The occurrence of two primary tumors in the nasopharynx is possible because it is primarily lined with stratified squamous epithelium and is rich in lymphoid cells. The residual tumor in our case may have been undifferentiated carcinoma following resolution of lymphoma after RCHOP treatment. We can only hypothesize the possibility of a collision tumor because it can only be diagnosed if the resected specimen showed different histological findings. Though collision tumors are very rare, their existence in the nasopharynx has yet to be reported. In the name of academic curiosity, we hope that this possibility heightens clinical awareness because recognition of such tumors is important as it will dictate appropriate treatment strategies, overall prognosis and survival rates.

\section{ACKNOWLEDGEMENT}

We acknowledge Ma. Karen A. Capuz, M.D., Rolando A. Lopez, M.D., and Alita B. Santos, M.D. for their support, literature contributions and advise in finishing this case report.

\section{REFERENCES}

1. Bahrami A, Truong LD, Ro JY. Undifferentiated tumor; true identity by immunohistochemistry. Arch Pathol Lab Med. 2008 Mar;132 (3):326-348.

2. Allam W, Ismaili N, Elmajjaoui S, Elgueddari B, Ismaili M, Errihani1 H. Primary Nasopharyngeal non-hodgkin lymphomas: a retrospective review of 26 Moroccan patients. BMC Ear Nose Throat Disord. 2009 Nov 17; 9:11.

3. Zong YS, Zhang RF, He SY, Qiu H. Histopathologic types and incidence of malignant nasopharyngeal tumors in Zhongshan County. Chin Med J (Engl). 1983 Jul;96(7):511 - 6.

4. Ensani F, Karimi SK. Nasopharyngeal Carcinoma: The role of immunohistochemistry in differentiation between undifferentiated carcinoma and malignant lymphoma: Report of 10 cases and review of literature. Acta Med Iran. 38 (1); 55-60: 2000.

5. Senba $M$, Zhong $X Y$, Itakura $H$. Immunohistochemical investigation of nasopharyngeal carcinoma using keratin, EMA, laminin, fibronectin, collagen type IV, laminin receptor, and laminin/collagen receptor antibodies. Acta Med Nagasaki.1993; 38(2): 182-185.

6. Gatter KC, Abdulaziz Z, Beverley P, Corvalan JR, Ford C, Lane EB. Use of monoclonal antibodies for the histopathological diagnosis of human malignancy. J Clin Pathol. 1982 Nov; 35(11): 125367

7. Gatter KC, Heryet A, Alock C, Mason DY. Clinical importance of analyzing malignant tumours of uncertain origin with immunohistological techniques. Lancet. 1985 Jun 8; 1(8441): 1302-5.

8. Michels S, Swanson PE, Frizzera G, Wick MR. Immunostaining for leukocyte common antigen using an amplified avidin-biotin-peroxidase complex method and paraffin sections: a study of 735 hematopoietic and nonhematopoietic human neoplasms. Arch Pathol Lab Med. 1987 Nov;111(11):1035-1039.

9. Raani P, Shasha Y, Perry C, Metser U, Naparstek E, Apter S, et al. Is CT scan still necessary for staging in Hodgkin and non-Hodgkin lymphoma patients in the PET/CT era? Ann Oncol. 2006 Jan; 17(1):117-122.

10. Sacchi S, Marcheselli L, Bari A, Marchesseli R, Pozzi S, Gobbi PG et al. Second malignancies after treatment of diffuse large B-cell non Hodgkin's lymphoma: a GISL cohort study. Haematologica 2008; 93:1335-1342.

11. Lasota J, Hyjek E, Koo CH, Blonski J, Miettinen M. Cytokeratin-positive large-cell lymphomas of B-cell lineage: A study of five phenotypically unusual cases verified by polymerase chain reaction. Am J Surg Pathol. 1996 Mar; 20(3): 346-354.

12. Tezer MS, Tuncel U, Ozlügedik S, Uzun M, Kulaçoglu S, Ünal A. Coexistence of laryngea squamous cell carcinoma and non-Hodgkin's lymphoma with nasopharyngeal involvement. $J$ Laryngol Otol .2006 Feb; 120(2):e2 1-4.

13. Brahmania M, Kanthan CS, Kanthan R. Collision tumor of the colon - colonic adenocarcinoma and ovarian granulosa cell tumor. World J Surg Oncol. 2007 Oct 20; 5:118.

14. Walvekar RR, Kane SV, D'Cruz AK. Collision tumor of the thyroid: follicular variant of papillary carcinoma and squamous carcinoma. World J Surg Oncol. 2006 Sep 19; 4: 65 